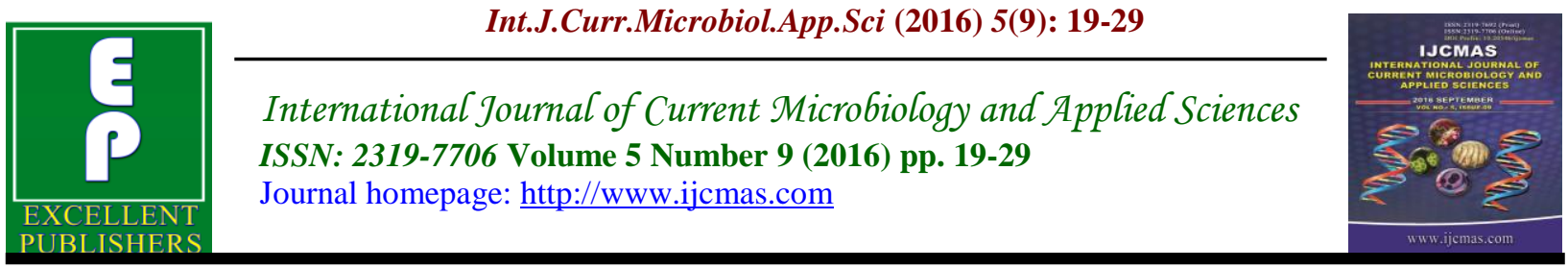

Original Research Article

http://dx.doi.org/10.20546/ijcmas.2016.509.003

\title{
Evaluation of Antifungal Activity of Artimesia, Litsea and Mikania Essential Oils against Post-harvested Fungal Diseases of Kiwifruits
}

\author{
Pramila Tripathi ${ }^{1}$, Habung Yami ${ }^{2}$ and A.K. Shukla ${ }^{3 *}$ \\ ${ }^{1}$ Department of Botany, DAV-PG College, Kanpur - 208001, India \\ ${ }^{2}$ Department of Botany, Rajiv Gandhi University, Itanagar - 791112, India \\ ${ }^{3}$ Department of Botany, Indira Gandhi National Tribal University, Amarkantak - 484887, India \\ *Corresponding author
}

\section{Keywords}

Essential oil, fungicidal, Phytopathogenic fungi, Artemesia, Litsea, Mikania.

\section{Article Info}

Accepted:

08 August 2016

Available Online:

10 September 2016

\section{A B S T R A C T}

Essential oils of plant species (Artemisia nilagerica, Litsea cubeba and Mikania cordata) were evaluate for their antifungal activity against phytopathogenic fungi viz. Alternaria alternata, Botrytis cinerea, Fusarium oxysporum and Penicillium expansum following Poisoned food technique method. Different concentration of oil such as $125 \mathrm{ppm}, 250 \mathrm{ppm}, 500 \mathrm{ppm}, 1000 \mathrm{ppm}$ and $5000 \mathrm{ppm}$ were taken to evaluate the effect. There was $100 \%$ inhibition in the growth of phytopathogenic fungi at 5000 and $1000 \mathrm{ppm}$ concentration by essential oil of Artemisia nilagerica and Litsea cubeba. The decrease in colony diameter or growth of fungus was corresponding to the concentration of oil. In comparison to others two, essential oil of Mikania cordata was less effective against phytopathogenic fungi. Minimum inhibitory concentration for oil of A. nilagerica and Litsea cubeba was found $500 \mathrm{ppm}$. At higher concentration essential oil of both species were reported fungicidal in nature. It can be stated on the basis of results that A. nilogirica and Litsea cubeba essential oil may be used as botanical pesticides for management of post harvest phytopathogenic fungal diseases. However, further investigations are needed in light of pharmacological tests.

\section{Introduction}

Kiwifruit (Actinidia deliciosa (A. Chev.) C.F. Liang and A.R. Ferguson var. deliciosa Hayward] is a climacteric and susceptible fruit to fungal decays in postharvest stage. After harvest, fruit rot diseases cause a severe loss of kiwifruit during cold storage, transportation, marketing, and in retail stores (Koh et al., 2003). Many fungi are associated with post harvest fruit rots of kiwifruit (Pennycook, 1985; Hawthorne et al., 1982). Botrytis gray mold rot caused by Botrytis cinerea is the most important and can directly invade the fruit or enter through wounds. Other fungal pathogens Phomopsis mali, Botryosphaeria dothidea and Diaporthe actinidiae have also been reported to cause post harvest fruit rots of kiwifruit (Koh et al., 2003). Post harvest application of dicarboximide fungicides immediately after harvest has been shown to 
reduce the incidence of rots to very low levels. However, this option is increasingly unacceptable due to public concern about and legislation on pesticide residues in fruit.

Many plant extracts have potential as natural antimicrobial agents that can be applied to agricultural produces, foods and pharmaceutics (Horburg, 1998; Maoz and Neeman, 1998) because they contain a phytochemical that exhibits antimicrobial and cytotoxic effects on microorganisms (Feldberg et al., 1988). Plants contain a wide range of bioactive secondary metabolites which include alkaloids, flavonoids, tannins, saponins, phenols, phlobatannins quinones, lecitins, polyphenols, glycosides, terpenoids, polypeptides and steroids (Edeoga et al., 2005; Enyiukwu and Awurum, 2013). Essential oils or volatile oils are very complex mixture of compounds whose constituents of the oils are mainly monoterpenes and sesquiterpenes. Generally, the action of essential oils is the result of the combined effect of both their active and inactive compounds (Tripathi and Shukla, 2007). Biologically active essential oils represent a rich potential source of an alternative and perhaps environmentally more acceptable disease management compounds (Tripathi and Shukla, 2009). With a broad range of natural fungicidal plant volatiles, numerous opportunities exist to explore their usefulness in controlling post-harvest diseases. Present study was aim to evaluate for antifungal activity of essential oils against phytopathogenic fungi of kiwifruits.

\section{Materials and Methods}

\section{Isolation and identification of phytopathogenic fungi}

Isolation of post harvest pathogens of kiwifruits were carried out from infected fruits on rose Bengal agar and potato dextrose agar medium (Johnson and Curl, 1972). Infected kiwifruits were randomly collected from market. Fruits were surface sterilized by $4 \%$ sodium hypochlorite and then by $75 \%$ alcohol and finally with sterilized distilled water. Small pieces of fruit were cut and placed in the petriplates containing sterilized medium and incubated at $27^{\circ} \mathrm{C}$ for $7-10$ days. Identification of fungal pathogens was done on the basis of morphological, cultural and microscopic characteristics as detailed in available literature (Barnett and Hunter, 1972; Domsch et al., 1980). In process of culture the isolated fungal pathogens were cultivated on Potato Dextrose Agar (PDA) medium and Peptone Dextrose Rose Bengal Agar medium were used. Potato dextrose agar medium (39 gm of Hi-PDA medium dissolved in $1000 \mathrm{ml}$ of distilled water) was used throughout the investigation. The medium was autoclaved and cooled to $40^{\circ} \mathrm{C}$ $\pm 2{ }^{\circ} \mathrm{C}$. Thirty milligram of streptomycin was added to it and mixed thoroughly so as to prevent bacterial contamination. Similarly Peptone Dextrose Rose Bengal Agar (31.55 gm of Hi-RBA medium dissolved in 1000 $\mathrm{ml}$ of distilled water) medium was prepared to maintain the fungal culture.

\section{Plant material collection and essential oils extraction}

Plants were collected from different parts of Arunachal Pradesh during the study period. Identification of plants was done by the plant taxonomist in the Department of Botany, Rajiv Gandhi University, Itanagar as well as by the Scientist from Regional Centre of Botanical Survey of India at Itanagar. Herbarium was preserved and voucher specimens were deposited in the department. Extraction of essential oils was carried out from some locally available larger number of angiospermic taxa namely 
Acorus calamus, Artimesia nilogerica, Erigeron canadensis, Eupatorium odoratum, Litsea cubeba, Mesua ferrae, Mikania cordata, Piper mullesua and Pogostemon cablin etc. Subsequently on getting results potent 3 plants were taken for detailed study. An amount of $250 \mathrm{gm}$ of fresh leaves of each plant were cut separately into small pieces and were thoroughly washed with sterilized water. The volatile fractions were isolated by hydro distillation through Clevenger's apparatus. Leaves of the plants were used for extraction of essential oils. The isolated fractions of plant parts exhibited two distinct layers an upper oily layer and the lower aqueous layer. Both the layers were separated and the essential oils were stored in clean glass vials after removing water traces with the help of capillary tubes and anhydrous sodium sulphate (Guenther, 1972).

\section{Antifungal activity assay}

Fungitoxic activities of the essential oils were tested by the poisoned food technique of Grover and Moore (1962) and Perrucci et al., (1994). Potato dextrose agar medium (39 gm of Hi-PDA medium dissolved in 1000 $\mathrm{ml}$ of distilled water) was used throughout the investigation. The medium was autoclaved and cooled to $40^{\circ} \mathrm{C} \pm 2^{\circ} \mathrm{C}$. Thirty milligram of streptomycin was added to it and mixed thoroughly so as to prevent bacterial contamination. A requisite amount of the oil was dissolved separately in $0.5 \mathrm{ml}$ of 0.01 percent of aqueous solution of Tween -80 in presterilized Petri plates $(7 \mathrm{~cm}$. diam.). While using Tween-80 as solvent care was taken in designing the experiments to evaluate the true effect of essential oils on the pathogenic fungi. PDA medium $(9.5 \mathrm{ml})$ was pipetted to each Petri plate and was mixed so as to obtain the requisite concentrations viz. 5000ppm, 1000ppm, $500 \mathrm{ppm}, 250 \mathrm{ppm}$ and $125 \mathrm{ppm}$. For control sets, requisite amount of sterilized water in place of the oil was added to the medium.

Discs of test fungi $(5 \mathrm{~mm}$ diam) were cut with the help of sterilized cork borer from the periphery of a seven day old culture and were inoculated aseptically to the center of each Petriplate of treatment and control sets. The petriplate were incubated at $27 \pm 1^{\circ} \mathrm{C}$ for six days in incubation chamber. Measurement of colony diameters of the test fungus in treatment and control sets were done in mutually perpendicular directions and were recorded in terms of percent mycelial inhibition using the following formula

Percentage of mycelial inhibition $=\frac{\mathrm{dc}-\mathrm{dt}}{\mathrm{dc}}$ Where

$\mathrm{dc}=$ mean colony diameter of control sets $\mathrm{dt}=$ mean colony diameter of treatment sets

Standardization of essential oils through fungitoxic properties

The standardization of essential oils was done through fungitoxic properties viz. minimum inhibitory concentration and nature of toxicity (Thompson, 1989).

\section{Minimum inhibitory concentration (MIC)}

To find out the minimum inhibitory concentration at which the oil showed absolute fungitoxicity (complete inhibition of growth of test fungi), experiments were carried out by the usual poisoned food technique. Different concentrations of the oils were prepared by dissolving separately their requisite amount in $0.5 \mathrm{ml}$ of 0.01 per cent of aqueous solution of Tween-80 and then mixing with $9.5 \mathrm{ml}$ potato dextrose agar medium. The medium of control sets 
contained requisite amount of sterilized water was added in $0.5 \mathrm{ml}$ Tween- 80 in place of oils. As usual the prepared plates were inoculated upside down aseptically with the assay disc of the test fungi to the center of petriplate of treatment and control sets. The petriplates were incubated at $27 \pm$ $1^{\circ} \mathrm{C}$ for six days in BOD incubator. Diameters of fungal colony of treatment and control sets were measured in mutually perpendicular directions on the seventh day and percentage inhibition calculated.

\section{Nature of toxicity}

Nature of toxicity (funtgistatic / fungicidal) of essential oils against the fungi was determined as suggested by Thompson (1989). Requisite amount of the oil was dissolved separately in $0.5 \mathrm{ml}$ of 0.01 per cent of aqueous solution of Tween-80 and mixed with $9.5 \mathrm{ml}$ potato dextrose agar medium to get final concentrations. Sterilized water was used in control sets in place of the oils. The plates were inoculated upside down aseptically with fungal disc (5mm diam.) taken from the periphery of a seven day old culture of the test fungi and were incubated for six days at $27 \pm 1^{\circ} \mathrm{C}$. On seventh day the inhibited discs were taken out from the plates, washed with sterilized water and reinoculated aseptically to plates containing fresh potato dextrose agar medium. The revival of the growth of the fungal discs was observed and the per cent inhibition of growth of the test fungi were calculated on the seventh day with respect to control sets.

\section{Results and Discussion}

\section{Evaluation of Essential Oils against Different Fungi}

Essential oils extracted from different plant species were evaluated to visualize their effect on the growth of four phytopathogenic fungi viz. Alternaria alternata, Botrytis cinerea, Fusarium oxysporum and Penicillium expansum following Poisoned food technique method. Different concentration of oil such as $125 \mathrm{ppm}$, 250ppm, 500ppm, 1000ppm and 5000ppm were taken to evaluate the effect. Simultaneously, a control was also maintained by inoculating culture disc on the medium without adding any oil.

\section{Artemisia nilagirica}

Essential oil of A. nilagirica inhibits the growth of all phytopathogenic fungi at higher concentration. At 5000ppm and $1000 \mathrm{ppm}$ concentrations $100 \%$ inhibition was recorded even after 15 days of incubation. The decrease in fungal growth was almost $50 \%$ even at 500ppm concentration of oil in case of all the tested fungus. Even at 125 and $250 \mathrm{ppm}$ concentration of oil growth was found inhibited. Slight expansion in diameter of fungus colony was recorded at lower concentration of oil however it always remains lesser than control. At higher concentration oil was found fungicidal in nature.

\section{Litsea cubeba}

Essential oil of Litsea cubeba inhibited the growth of all phytopathogenic fungi. The decrease in colony diameter or growth of fungus was corresponding to the concentration of oil. On $F$. oxysporum effect of oil was also found to be sever and $100 \%$ inhibition was observed at 5000ppm concentration and at $1000 \mathrm{ppm}$ slight growth was seen on the $15^{\text {th }}$ day of observation. In case of B. cinerea, effect of $L$. cubeba essential oil was quite remarkable, at 5000 and 1000ppm concentration 100\% inhibition was recorded even after 15 days of incubation. Essential oil of L. cubeba shows drastic effect on the growth of fungus 
colony at higher concentration 5000 and $1000 \mathrm{ppm}$. At lower level of concentration i,e 125,250 , and $500 \mathrm{ppm}$ of oil colony growth was recorded for all the four phytopathogenic fungi but it always remain lesser than the control. The effect of essential oil was corresponding to their concentration.

\section{Mikania cordata}

Essential oil of $M$. cordata inhibits the growth of all four phytopathogenic fungi. Hundred percent of inhibition in growth of tested fungus was not found at any concentration of oil. However, it was observed that growth was reduced on increasing the concentration in case of all the tested phytopathogenic fungi. Comparatively $P$. expansum, A. alternata and $B$. cinerea growth was effected more than the $F$. oxysporum. At the end of study period growth of $F$. oxysporum at lower concentration of treatment was similar to control.

\section{Minimum Inhibitory concentration (MIC)}

Inhibitory evaluation of essential oils against phytopathogenic fungi showed the effective results. Essential oil of $A$. nilagirica and $L$. cubeba were found fungi toxic at 250ppm for all the tested fungus. The essential oil of Artemisia nilagirica and L. cubeba were found fungicidal at 5000ppm and 1000ppm concentration and minimum inhibitory contration was reported 500ppm. The essential oil of Mikania cordata was inhibitory at higher concentration for all the phytopathogenic fungi.

\section{Nature of toxicity}

A. nilagirica and L. cubeba oils were found fungicidal for all the phytopathogenic fungi. At lower concentrations oils were fungistatic. Whereas, Mikania cordata oil reported fungistatic in nature.

Essential oil of $A$. nilagerica inhibits the growth of all phytopathogenic fungi at higher concentration. At 5000ppm and $1000 \mathrm{ppm}$ concentrations $100 \%$ inhibition was recorded even after 15 days of incubation. The decrease in colony diameter or growth of fungus was corresponding to the concentration of oil. At low concentration nature of oil was fungistatic.

Sati et al., (2012) reported that essential oil contained approximately $\quad 79.91 \%$ monoterpenoids and $18.25 \%$ sesquiterpenoids. Thujone (36.35\%), thujone $(9.37 \%)$, germacrene D $(6.32 \%)$, 4terpineol $(6.31 \%)$, -caryophyllene $(5.43 \%)$, camphene $(5.47 \%)$ and borneol $(4.12 \%)$ as the major constituents. The essential oil exhibited significant antifungal activity against Rhizoctonia solani (ED50, $85.75 \mathrm{mg}$ L1), Sclerotium rolfsii (ED50, $87.63 \mathrm{mg} \mathrm{L1)}$ and Macrophomina phaseolina (ED50, 93.23 mg L1). Padalia et al., (2014) found that essential oils were mainly composed of monoterpenoids $\quad(59.0 \%-77.3 \%) \quad$ and sesquiterpenoids $(15.7 \%-31.6 \%)$.

The major constituents identified were artemisia ketone (38.3\%-61.2\%), chrysanthenone (1.5\%-7.7\%), germacrene D (3.1\%-6.8\%), $\beta$-caryophyllene (1.9\%-6.8\%), germacra-4,5, 10-trien-1- $\alpha$-ol (1.9\%-4.9\%) and artemisia alcohol (1.4\%-3.6\%). Stappen et al., (2014) reported that A. nilagirica essential oil have nonselective antifungal activity against plant pathogens Colletotrichum acutatum, Colletotrichum fragariae and Colletotrichum gloeosporioides. Presence of terpenoides in large quantity and other compounds in small quantity would have perhaps played antifungal property against the phytopathogenic fungi. 
Table.1 Effect of Artemisia nilagerica essential oil on the phytopathogenic fungi

\begin{tabular}{|c|c|c|c|c|c|c|}
\hline Period & \multicolumn{6}{|c|}{ Alternaria alternate } \\
\hline Days & 5000ppm & 1000ppm & 500ppm & 250ppm & 125ppm & Control \\
\hline $5^{\text {th }}$ & $0.00 \pm 0.00$ & $0.00 \pm 0.00$ & $2.60 \pm 0.20$ & $3.35 \pm 0.05$ & $3.90 \pm 0.10$ & $4.50 \pm 0.10$ \\
\hline $7^{\text {th }}$ & $0.00 \pm 0.00$ & $0.00 \pm 0.00$ & $3.25 \pm 0.55$ & $3.05 \pm 0.05$ & $4.50 \pm 0.10$ & $5.90 \pm 0.00$ \\
\hline $9^{\text {th }}$ & $0.00 \pm 0.00$ & $0.00 \pm 0.00$ & $3.25 \pm 0.55$ & $4.15 \pm 0.05$ & $5.15 \pm 0.15$ & $6.50 \pm 0.10$ \\
\hline $11^{\text {th }}$ & $0.00 \pm 0.00$ & $0.00 \pm 0.00$ & $3.25 \pm 0.55$ & $5.00 \pm 0.10$ & $5.25 \pm 0.25$ & $6.80 \pm 0.10$ \\
\hline $13^{\text {th }}$ & $00 \pm 0.00$ & $0.00 \pm 0.00$ & $3.30 \pm 0.50$ & $5.00 \pm 0.10$ & $5.60 \pm 0.20$ & $6.80 \pm 0.10$ \\
\hline \multirow[t]{2}{*}{$15^{\text {th }}$} & $0.00 \pm 0.00$ & $0.00 \pm 0.00$ & $3.30 \pm 0.50$ & $5.00 \pm 0.10$ & $5.60 \pm 0.20$ & $7.00 \pm 0.10$ \\
\hline & \multicolumn{6}{|c|}{ Botrytis cinerea } \\
\hline Days & 5000ppm & 1000ppm & 500ppm & 250ppm & & Control \\
\hline 5 th & $0.00 \pm 0.00$ & $0.00 \pm 0.00$ & $2.6 \pm 0.25$ & $3.35 \pm 0.05$ & & $4.50 \pm 0.10$ \\
\hline 7th & $0.00 \pm 0.00$ & $0.00 \pm 0.00$ & 3.25 & $4.05 \pm 0.20$ & & \pm 0.00 \\
\hline 9th & $00 \pm 0.00$ & $.00 \pm 0.00$ & $3.25 \pm 0$ & $4.15 \pm 0.10$ & & $=0.10$ \\
\hline 11th & $00 \pm 0.00$ & $.00 \pm 0.00$ & $3.25 \pm 0.6$ & $5.00 \pm 0$ & $5.25 \pm 0.20$ & $6.80 \pm 0.10$ \\
\hline 13th & $00 \pm 0.00$ & $0.00 \pm 0.00$ & $3.3 \pm 0$ & $5.00 \pm 0$ & $5.60 \pm 0.20$ & $6.80 \pm 0.10$ \\
\hline \multirow[t]{2}{*}{15 th } & $0.00 \pm 0.00$ & $0.00 \pm 0.00$ & $3.3 \pm$ & $5.00 \pm 0.30$ & & $7.00 \pm 0.10$ \\
\hline & \multicolumn{6}{|c|}{ Fusarium oxysporum } \\
\hline Days & & & $500 \mathrm{p}$ & 250pp & & \\
\hline $5^{\text {th }}$ & $0.00 \pm 0.00$ & $0.00 \pm 0.00$ & $1.75 \pm 0.05$ & $2.00 \pm 0.00$ & $2.10 \pm 0.10$ & $4.00 \pm 0.00$ \\
\hline $7^{\text {th }}$ & $0.00 \pm 0.00$ & $0.00 \pm 0.00$ & $2.15 \pm 0.05$ & $2.35 \pm 0.05$ & $2.50 \pm 0.10$ & $4.50 \pm 0.10$ \\
\hline $9^{\text {th }}$ & $0.00 \pm 0.00$ & $0.00 \pm 0.00$ & $2.45 \pm 0.05$ & $2.75 \pm 0.05$ & $2.90 \pm 0.10$ & $4.70 \pm 0.10$ \\
\hline 11th & $0.00 \pm 0.00$ & $0.00 \pm 0.00$ & $2.90 \pm 0.00$ & $2.75 \pm 0.05$ & $2.90 \pm 0.10$ & $5.40 \pm 0.10$ \\
\hline $13^{\text {th }}$ & $0.00 \pm 0.00$ & $0.00 \pm 0.00$ & $3.35 \pm 0.05$ & $3.50 \pm 0.00$ & $3.60 \pm 0.10$ & $6.30 \pm 0.10$ \\
\hline \multirow[t]{2}{*}{$15^{\text {th }}$} & $0.00 \pm 0.00$ & $0.00 \pm 0.00$ & $3.35 \pm 0.05$ & $4.00 \pm 0.00$ & $4.25 \pm 0.25$ & $7.00 \pm 0.10$ \\
\hline & \multicolumn{6}{|c|}{ Penicillium expensum } \\
\hline Days & & & 500ppm & 250ppm & & Control \\
\hline 5 th & $0.00 \pm 0.00$ & $0.00 \pm 0.00$ & $1.20 \pm 0.10$ & $3.40 \pm 0.20$ & $3.75 \pm 0.05$ & $4.50 \pm 0.10$ \\
\hline 7th & $0.00 \pm 0.00$ & $0.00 \pm 0.00$ & $2.05 \pm 0.05$ & $3.80 \pm 0.20$ & $4.00 \pm 0.00$ & $5.90 \pm 0.00$ \\
\hline 9th & $0.00 \pm 0.00$ & $0.00 \pm 0.00$ & $2.55 \pm 0.25$ & $4.10 \pm 0.10$ & $4.35 \pm 0.05$ & $6.50 \pm 0.10$ \\
\hline 11th & $0.00 \pm 0.00$ & $0.00 \pm 0.00$ & $2.55 \pm 0.25$ & $4.10 \pm 0.10$ & $5.05 \pm 0.05$ & $6.80 \pm 0.10$ \\
\hline 13th & $0.00 \pm 0.00$ & $0.00 \pm 0.00$ & $2.75 \pm 0.25$ & $4.35 \pm 0.15$ & $5.05 \pm 0.05$ & $6.80 \pm 0.10$ \\
\hline 15 th & $0.00 \pm 0.00$ & $0.00 \pm 0.00$ & $2.75 \pm 0.25$ & $4.35 \pm 0.15$ & $5.15 \pm 0.05$ & $7.00 \pm 0.10$ \\
\hline
\end{tabular}


Table.2 Effect of Litsea cubeba essential oil on the phytopathogenic fungi.

\begin{tabular}{|c|c|c|c|c|c|c|}
\hline Period & \multicolumn{6}{|c|}{ Alternaria alternate } \\
\hline Days & 5000ppm & 1000ppm & 500ppm & 250ppm & 125ppm & Control \\
\hline $5^{\text {th }}$ & $0.00 \pm 0.00$ & $0.00 \pm 0.00$ & $0.80 \pm 0.00$ & $1.10 \pm 0.60$ & $2.20 \pm 0.10$ & $4.50 \pm 0.10$ \\
\hline $7^{\text {th }}$ & $0.00 \pm 0.00$ & $0.00 \pm 0.00$ & $0.80 \pm 0.00$ & $2.90 \pm 0.50$ & $2.65 \pm 0.15$ & $5.90 \pm 0.00$ \\
\hline $9^{\text {th }}$ & $0.00 \pm 0.00$ & $0.00 \pm 0.00$ & $1.20 \pm 0.10$ & $3.05 \pm 0.45$ & $3.65 \pm 0.55$ & $6.50 \pm 0.10$ \\
\hline $11^{\text {th }}$ & $0.00 \pm 0.00$ & $0.00 \pm 0.00$ & $1.50 \pm 0.10$ & $3.05 \pm 0.45$ & $3.65 \pm 0.55$ & $6.80 \pm 0.10$ \\
\hline $13^{\text {th }}$ & $00 \pm 0.00$ & $.00 \pm 0.00$ & $1.90 \pm 0.05$ & $3.05 \pm 0.45$ & $3.65 \pm 0.55$ & $6.80 \pm 0.10$ \\
\hline \multirow[t]{2}{*}{$15^{\text {th }}$} & $0.00 \pm 0.00$ & $0.00 \pm 0.00$ & $1.90 \pm 0.05$ & $3.05 \pm 0.45$ & $3.65 \pm 0.55$ & $7.00 \pm 0.10$ \\
\hline & \multicolumn{6}{|c|}{ Botrytis cinerea } \\
\hline Days & 5000ppm & 1000ppm & 500ppm & 250ppm & 125ppm & Control \\
\hline $5^{\text {th }}$ & $0.00 \pm 0.00$ & $0.00 \pm 0.00$ & $2.25 \pm 0.25$ & $3.35 \pm 0.15$ & $4.25 \pm 0.15$ & $4.50 \pm 0.10$ \\
\hline $7^{\text {th }}$ & $0.00 \pm 0.00$ & $0.00 \pm 0.00$ & $4.90 \pm 0.30$ & $5.75 \pm 0.25$ & $5.05 \pm 0.95$ & $5.90 \pm 0.00$ \\
\hline $9^{\text {th }}$ & $.00 \pm 0.00$ & $0.00 \pm 0.00$ & $5.60 \pm 0.40$ & $5.90 \pm 0.10$ & $5.25 \pm 1.05$ & $6.50 \pm 0.10$ \\
\hline $11^{\text {th }}$ & $.00 \pm 0.00$ & $0.00 \pm 0.00$ & $6.10 \pm 0.10$ & $6.65 \pm 0.35$ & $7.00 \pm 0.00$ & $6.80 \pm 0.10$ \\
\hline $13^{\text {th }}$ & $.00 \pm 0.00$ & $0.00 \pm 0.00$ & $6.20 \pm 0.20$ & $6.75 \pm 0.25$ & $7.00 \pm 0.00$ & $6.80 \pm 0.10$ \\
\hline \multirow[t]{2}{*}{$15^{\text {th }}$} & $0.00 \pm 0.00$ & $0.00 \pm 0.00$ & $6.20 \pm 0.20$ & $6.75 \pm 0.25$ & $7.00 \pm 0.00$ & $7.00 \pm 0.10$ \\
\hline & \multicolumn{6}{|c|}{ Fusarium oxysporum } \\
\hline Days & & & & 250 & $125 p$ & Control \\
\hline $5^{\text {th }}$ & $0.00 \pm 0.00$ & & & $2.45 \pm 0.05$ & $3.30 \pm 0.10$ & $4.00 \pm 0.00$ \\
\hline $7^{\text {th }}$ & $0.00 \pm 0.00$ & $0.00 \pm 0.00$ & $1.40 \pm 0.00$ & $4.20 \pm 1.00$ & $5.00 \pm 1.00$ & $4.50 \pm 0.10$ \\
\hline $9^{\text {th }}$ & $0.00 \pm 0.00$ & $0.00 \pm 0.00$ & $1.60 \pm 0.00$ & $4.75 \pm 1.25$ & $5.00 \pm 1.00$ & $4.70 \pm 0.10$ \\
\hline $11^{\text {th }}$ & $0.00 \pm 0.00$ & $0.00 \pm 0.00$ & $1.90 \pm 0.10$ & $5.25 \pm 0.75$ & $5.00 \pm 1.00$ & $5.40 \pm 0.10$ \\
\hline $13^{\text {th }}$ & $0.00 \pm 0.00$ & $0.00 \pm 0.00$ & $2.00 \pm 0.00$ & $5.85 \pm 0.15$ & $5.05 \pm 0.95$ & $6.30 \pm 0.10$ \\
\hline \multirow[t]{2}{*}{$15^{\text {th }}$} & $0.00 \pm 0.00$ & $0.00 \pm 0.00$ & $2.20 \pm 0.10$ & $6.00 \pm 0.00$ & $5.20 \pm 1.00$ & $7.00 \pm 0.10$ \\
\hline & \multicolumn{6}{|c|}{ Penicillium expensum } \\
\hline Days & 5000ppm & $1000 \mathrm{ppm}$ & 500ppm & 250ppm & 125ppm & Control \\
\hline $5^{\text {th }}$ & $0.00 \pm 0.00$ & $0.00 \pm 0.00$ & $1.45 \pm 0.25$ & $2.25 \pm 0.15$ & $1.95 \pm 0.35$ & $4.00 \pm 0.00$ \\
\hline $7^{\text {th }}$ & $0.00 \pm 0.00$ & $0.00 \pm 0.00$ & $2.35 \pm 0.25$ & $2.5 \pm 0.40$ & $2.55 \pm 0.85$ & $4.50 \pm 0.10$ \\
\hline $9^{\text {th }}$ & $0.00 \pm 0.00$ & $0.00 \pm 0.00$ & $2.80 \pm 0.60$ & $3.35 \pm 0.15$ & $2.55 \pm 0.85$ & $4.70 \pm 0.10$ \\
\hline $11^{\text {th }}$ & $0.00 \pm 0.00$ & $0.00 \pm 0.00$ & $3.15 \pm 0.75$ & $3.65 \pm 0.45$ & $2.55 \pm 0.85$ & $5.40 \pm 0.10$ \\
\hline $13^{\text {th }}$ & $0.00 \pm 0.00$ & $0.00 \pm 0.00$ & $3.50 \pm 0.90$ & $4.00 \pm 0.70$ & $2.60 \pm 0.80$ & $6.30 \pm 0.10$ \\
\hline $15^{\text {th }}$ & $0.00 \pm 0.00$ & $0.00 \pm 0.00$ & $4.05 \pm 0.55$ & $4.05 \pm 0.75$ & $2.70 \pm 0.80$ & $7.00 \pm 0.10$ \\
\hline
\end{tabular}


Table.3 Effect of Mikania cordata essential oil on the phytopathogenic fungi

\begin{tabular}{|c|c|c|c|c|c|c|}
\hline Period & \multicolumn{6}{|c|}{ Alternaria alternate } \\
\hline Days & 5000ppm & 1000ppm & $500 \mathrm{ppm}$ & 250ppm & 125ppm & Control \\
\hline $5^{\text {th }}$ & $1.90 \pm 0.10$ & $2.10 \pm 0.10$ & $2.25 \pm 0.05$ & $2.35 \pm 0.05$ & $2.50 \pm 0.10$ & $4.50 \pm 0.10$ \\
\hline $7^{\text {th }}$ & $2.20 \pm 0.10$ & $2.65 \pm 0.15$ & $2.95 \pm 0.35$ & $3.35 \pm 0.05$ & $3.35 \pm 0.35$ & $5.90 \pm 0.00$ \\
\hline $9^{\text {th }}$ & $3.10 \pm 0.20$ & $3.30 \pm 0.40$ & $4.00 \pm 1.31$ & $4.05 \pm 0.15$ & $5.20 \pm 0.00$ & $6.50 \pm 0.10$ \\
\hline $11^{\text {th }}$ & $3.40 \pm 0.20$ & $3.70 \pm 0.50$ & $4.15 \pm 1.45$ & $5.20 \pm 0.00$ & $5.55 \pm 0.05$ & $6.80 \pm 0.10$ \\
\hline $13^{\text {th }}$ & $3.55 \pm 0.05$ & $4.10 \pm 0.70$ & $4.15 \pm 1.45$ & $5.65 \pm 0.05$ & $5.80 \pm 0.10$ & $6.80 \pm 0.10$ \\
\hline \multirow[t]{2}{*}{$15^{\text {th }}$} & $3.70 \pm 0.20$ & $4.10 \pm 0.70$ & $4.15 \pm 1.45$ & $5.65 \pm 0.05$ & $5.80 \pm 0.10$ & $7.00 \pm 0.10$ \\
\hline & \multicolumn{6}{|c|}{ Botrytis cinerea } \\
\hline Days & 5000ppm & 1000ppm & 500ppm & 250ppm & 125ppm & Control \\
\hline $5^{\text {th }}$ & $1.55 \pm 0.05$ & $2.60 \pm 0.10$ & $2.90 \pm 0.00$ & $3.05 \pm 0.05$ & $3.05 \pm 0.15$ & $4.50 \pm 0.10$ \\
\hline $7^{\text {th }}$ & $2.20 \pm 0.30$ & $3.50 \pm 0.50$ & $4.05 \pm 0.15$ & $4.35 \pm 0.05$ & $4.00 \pm 0.40$ & $5.90 \pm 0.00$ \\
\hline $9^{\text {th }}$ & $3.30 \pm 1.30$ & $5.50 \pm 0.30$ & $5.55 \pm 0.45$ & $6.40 \pm 0.00$ & $4.10 \pm 0.50$ & $6.50 \pm 0.10$ \\
\hline $11^{\text {th }}$ & $3.50 \pm 1.50$ & $5.70 \pm 0.10$ & $5.90 \pm 0.20$ & $6.40 \pm 0.00$ & $4.15 \pm 0.45$ & $6.80 \pm 0.10$ \\
\hline $13^{\text {th }}$ & $3.65 \pm 1.65$ & $5.85 \pm 0.15$ & $5.90 \pm 0.20$ & $6.40 \pm 0.00$ & $4.15 \pm 0.45$ & $6.80 \pm 0.10$ \\
\hline \multirow[t]{2}{*}{$15^{\text {th }}$} & $3.90 \pm 1.90$ & $5.90 \pm 0.10$ & $5.90 \pm 0.20$ & $6.40 \pm 0.00$ & $4.15 \pm 0.45$ & $7.00 \pm 0.10$ \\
\hline & \multicolumn{6}{|c|}{ Fusarium oxysporum } \\
\hline Days & 5000ppm & 1000ppm & $500 \mathrm{pl}$ & 250p & 125ppm & Control \\
\hline $5^{\text {th }}$ & $2.80 \pm 0.00$ & $3.50 \pm 0.05$ & $3.50 \pm 0.05$ & $3.00 \pm 0.00$ & $2.90 \pm 0.10$ & $4.00 \pm 0.00$ \\
\hline $7^{\text {th }}$ & $4.20 \pm 0.00$ & $4.50 \pm 0.00$ & $4.65 \pm 0.05$ & $4.60 \pm 0.00$ & $3.75 \pm 0.65$ & $4.50 \pm 0.10$ \\
\hline $9^{\text {th }}$ & $6.50 \pm 0.05$ & $5.80 \pm 0.30$ & $6.50 \pm 0.00$ & $6.25 \pm 0.25$ & $4.10 \pm 0.90$ & $4.70 \pm 0.10$ \\
\hline $11^{\text {th }}$ & $6.85 \pm 0.05$ & $6.00 \pm 0.50$ & $6.75 \pm 0.05$ & $6.50 \pm 0.50$ & $4.85 \pm 1.65$ & $5.40 \pm 0.10$ \\
\hline $13^{\text {th }}$ & $6.95 \pm 0.05$ & $6.15 \pm 0.65$ & $6.90 \pm 0.00$ & $6.50 \pm 0.50$ & $5.15 \pm 1.85$ & $6.30 \pm 0.10$ \\
\hline \multirow[t]{2}{*}{$15^{\text {th }}$} & $6.95 \pm 0.05$ & $6.15 \pm 0.65$ & $7.00 \pm 0.00$ & $6.50 \pm 0.50$ & $5.15 \pm 1.85$ & $7.00 \pm 0.10$ \\
\hline & \multicolumn{6}{|c|}{ Penicillium expensum } \\
\hline Days & 5000ppm & 1000ppm & 500ppm & 250ppm & 125ppm & Control \\
\hline $5^{\text {th }}$ & $1.65 \pm 0.05$ & $1.90 \pm 0.10$ & $2.05 \pm 0.05$ & $2.25 \pm 0.05$ & $2.35 \pm 0.05$ & $4.50 \pm 0.10$ \\
\hline $7^{\text {th }}$ & $2.25 \pm 0.05$ & $2.45 \pm 0.35$ & $2.95 \pm 0.35$ & $2.95 \pm 0.35$ & $3.35 \pm 0.05$ & $5.90 \pm 0.00$ \\
\hline $9^{\text {th }}$ & $3.10 \pm 0.20$ & $3.25 \pm 0.65$ & $3.65 \pm 1.05$ & $4.00 \pm 1.30$ & $5.20 \pm 0.10$ & $6.50 \pm 0.10$ \\
\hline $11^{\text {th }}$ & $3.40 \pm 0.20$ & $3.40 \pm 0.80$ & $3.80 \pm 1.20$ & $4.15 \pm 1.45$ & $5.55 \pm 0.05$ & $6.80 \pm 0.10$ \\
\hline $13^{\text {th }}$ & $3.55 \pm 0.05$ & $3.70 \pm 1.10$ & $3.85 \pm 1.25$ & $4.15 \pm 1.45$ & $5.80 \pm 0.10$ & $6.80 \pm 0.10$ \\
\hline $15^{\text {th }}$ & $3.70 \pm 0.20$ & $3.70 \pm 1.10$ & $3.90 \pm 1.30$ & $4.15 \pm 1.45$ & $5.80 \pm 0.10$ & $7.00 \pm 0.10$ \\
\hline
\end{tabular}


Table.4 Minimum inhibitory concentration of essential oils against pathogenic fungi

\begin{tabular}{|l|l|l|l|l|}
\hline MIC of oils against fungi \\
\hline \multirow{2}{*}{$\begin{array}{l}\text { Essential oils of } \\
\text { plants }\end{array}$} & Phytopathogenic fungi \\
\cline { 2 - 5 } & A. alternata & B. cinerea & F. oxysporum & P. expansum \\
\hline Artemisia nilagerica & $500 \mathrm{ppm}$ & $500 \mathrm{ppm}$ & $500 \mathrm{ppm}$ & $500 \mathrm{ppm}$ \\
\hline Litsea cubeba & $500 \mathrm{ppm}$ & $500 \mathrm{ppm}$ & $500 \mathrm{ppm}$ & $500 \mathrm{ppm}$ \\
\hline Mikania cordata & Higher Conc. & Higher Conc. & Higher Conc. & Higher Conc. \\
\hline
\end{tabular}

Table.5 Toxicity nature of Essential oils on phytopathogenic fungi

\begin{tabular}{|l|l|l|l|l|}
\hline Essential oils & A. alternata & B. cinerea & F. oxysporum & P. expansum \\
\hline Artemisia nilagerica & $\begin{array}{l}\text { Fungicidal at } \\
1000 \mathrm{ppm}\end{array}$ & $\begin{array}{l}\text { Fungicidal at } \\
1000 \mathrm{ppm}\end{array}$ & $\begin{array}{l}\text { Fungicidal at } \\
1000 \mathrm{ppm}\end{array}$ & $\begin{array}{l}\text { Fungicidal at } \\
1000 \mathrm{ppm}\end{array}$ \\
\hline Litsea cubeba & $\begin{array}{l}\text { Fungicidal at } \\
1000 \mathrm{ppm}\end{array}$ & $\begin{array}{l}\text { Fungicidal at } \\
1000 \mathrm{ppm}\end{array}$ & $\begin{array}{l}\text { Fungicidal at } \\
1000 \mathrm{ppm}\end{array}$ & $\begin{array}{l}\text { Fungicidal at } \\
1000 \mathrm{ppm}\end{array}$ \\
\hline Mikania cordata & Fungistatic & Fungistatic & Fungistatic & Fungistatic \\
\hline
\end{tabular}

Growth of P. expansum, F. oxysporum and A. alternata was $100 \%$ inhibited at 5000ppm. Si et al., (2012) reported 59 compounds from L. cubeba oil out of which dominant components were monoterpenes (94.4-98.4\%), represented mainly by neral and geranial (78.7-87.4\%), and D-Limonene was in lesser constituent $(0.7-5.3 \%)$. Several components were only detected in certain regions and compounds such as ocymene and eremophilene have never before been reported in EOLC. Su et al., (2012) identified main components in oil as $\beta$ caryophyllene $(13.0 \%), \tau$-cadinol $(11.1 \%)$, $\alpha$-cadinol (8.6\%), $\alpha$-humulene (7.5\%), $\alpha$ pinene $(7.0 \%)$, globulol $(6.6 \%)$, and $\beta$ eudesmol $(6.1 \%)$. The anti-wood-decay fungal activity of the oil showed that the oil was inhibitory for wood-decay-fungi species and compounds were determined to be $\tau$ cadinol, $\alpha$-cadinol, and $\beta$-eudesmol. Yang (2010) through preliminary bioassay study showed L. cubeba oil has good fungicidal activities against Sclerotinia sclerotiorum, Thanatephorus cucumeris, Pseudocercospora musae and Colletotrichum gloeosporioides at the concentration of 588 and $272 \mu \mathrm{M}$, and the essential oil has good fungicidal activities against $T$. cucumeris and $S$. sclerotiorum, with $\mathrm{IC}_{50}$ values of 115.58 and $151.25 \mu \mathrm{g} / \mathrm{mL}$, repectively.

Essential oil of Mikania cordata inhibits the growth of all four phytopathogenic fungi. Hundred percent of inhibition in growth of tested fungus was not found at any concentration of oil. Comparatively $P$. expansum, $A$. alternata and $B$. cinerea growth was effected more than the $F$. oxysporum. At the end of study period growth of $F$. oxysporum at lower concentration of treatment was similar to control. Mikania cordata essential oil consist chemical groups like coumarins and derivatives, sesquiterpenes, sesquiterpenes lactones, diterpenes, phytosterols/terpenoids and flavonoids (Rufatto et al., 2012). Many authors attributed the pharmacological effect of guaco to coumarin (1, 2-benzopirone) (Santos et al., 2006). However, our results made clear that this is not the only bioactive component present in the extracts we tested. 
Ethyl acetate is a solvent capable of extract coumarin and several terpenes and phenolic compounds, which have a wide range of biological activities. These include plant growth-regulators, secondary compounds against insects, and anti-bacterial properties (Huang et al., 2009). Some flavonoids and dicaffeoylquinic acid butyl esters have been recently described as bioactive for $M$. micrantha (Wei et al., 2004).

It can be stated on the basis of results that $A$. nilogirica and Litsea cubeba essential oil may be used as botanical pesticides for management of post harvest phytopathogenic fungal diseases. However, further investigations are needed in light of pharmacological tests.

\section{References}

Barnett, B.B. and Hunter, H.L. 1972. Illustrated genera of imperfect fungi. Burgess Publishing Co. Minneapolis, USA.

Domsch, K.H., Games, W. and Anderson, T. 1980. Compendium of soil fungi. Academic Press, London.

Edeoga, H.O., Okwu, D.E. and Mbaebie, B.O. 2005. Phytochemical constituents of some Nigerian medicinal plants. Afr. J. Biotechnol., 4: 685-688.

Enyiukwu, D.N., Awurum, A.N. 2013. Fungitoxic principles and antifungal activity of extracts from Carica papaya and Piper guineense on Colletotrichum destructivum. Cont. J. Biol. Sci., 7: 29-36.

Grover, R.K., Moore, J.D. 1962. Toximetric study of fungicides against brown rot organism,Sclerotinia fructicola and S.laxa.Phytopathol., 52: 876-880.

Guenther, E. 1972. In The essential oils Vol.I and IV. Robert, E. Krieger publishing Co. Hutington, New York.

Hawthorne, B.T., Rees-George, J., Samuels,
G.J. 1982. Fungi associated with leaf spots and postharvest fruit rots of "kiwifruit" (Actinidia chinensis) in New Zealand. New Zealand J. Bot., 20: 143-150.

Horburg, H. 1998. Influence of volatile extracts on storage pathogen of carrots in vitro. Vaxtskyddsnotise, 64: 87-89.

Huang, H., Ye, W., Wei, X an. Zhang, C 2009. Allelopathic potential of sesquiterpene lactones and phenolic constituents from Mikania micrantha H. B. K. Biochemical Systematics and Ecol., 36: 867-871.

Johnson, L.E. and Curl, E.A. 1972. Methods for research on ecology of soil borne plant pathogens. Burgess Publishing Co. Minneapolis, USA.

Koh, Y.J., Jung, J.S., Hur, J.S. 2003. Current status of occurrence of major diseases on kiwifruits and their control in Korea. Acta Horticulturae, 61: 437443.

Maoz, M., and Neeman, I. 1998. Antimicrobial effects of aqueous plant extracts on the fungi Microsporum canis and Trichophyton rubrum and on three bacteria species. Lett. Appl. Microbiol., 26: 61-63.

Pennycook, S.R. 1985. Fungal fruit rots of Actinidia deliciosa (kiwifruit). New Zealand J. Experimental Agri., 13: 289-299.

Perrucci, S., Mancianti, f., Ciont, P.L., Flamini, G., Morelli, I., Macchioni, G. 1994. In vitro antifungal activity of essential oils against some isolates of Microsporum canis and M.gypseum. Planta. Medi, 60: 184-187.

Rufatto, L.C., Gower, A., Schwambach, J and Moura, S. 2012. Genus Mikania: chemical composition and phytotherapeutical activity. Rev. bras. Farmacogn, 22: 6-10.

Santos, S.C., Krueger, C.L., Steil, A.A., Krueger, M.R., Biavati, M.Wand 
Wisnievski-Junior, A. 2006. LC characterization of guaco medicinal extracts, Mikania laevigata and $M$. glomerata, and their effects on allergic pneumonitis. Planta Med., 72: 679684.

Sati, S.C., Sati, N., Ahluwalia, V., Walia, S and Sati, O.P. 2012. Chemical composition and antifungal activity of Artemisia nilagirica essential oil growing in northern hilly areas of India. Natural Product Res., 27: 45-48

Si, L., Chen, Y., Han, X., Zhan, Z., Tian, S., Cui, Q and Wang, Y. 2012. Chemical Composition of Essential Oils of Litsea cubeba Harvested from Its Distribution Areas in China. Mol., 17: 7057-7066.

Stappen, I., Wanner, J., Tabanca, N., Wedge, D.E,. Ali, A., Khan, I.A., Kaul, V.K., Lal, B., Jaitak, V., Gochev, V., Girova, T., Stoyanova, A., Schmidt, E., Jirovetz, L. 2014. Chemical Composition and Biological Effects of Artemisia maritima and Artemisia nilagirica Essential Oils from Wild Plants of Western Himalaya. Planta Med., 80: 1079-87
Su, Y.C., Ho, C.L. 2012. Composition and Two Activities of the Leaf Essential Oil of Litsea acuminata (Blume) Kurata from Taiwan. Rec. Nat. Prod., 7: 27-34

Thompson, D.P. 1989. Fungitoxic activity of essential oil components on food storage fungi. Mycologia, 81: 151-153.

Tripathi, P. and Shukla, A.K. 2007. Emerging non conventional technologies for control of post harvest diseases of perishables. Fresh Produce, 1: 111-120.

Tripathi, P. and Shukla, A.K. 2009. Application of essential oils for control of stem end rot of mango fruits during storage. Int. J. Post Harvest Technol. Innovations, 1: 405-415.

Yang, Y., Jiang, J., Qunei, L., Yan, X., Zhao, J., Yuan, H., and Wang, M. 2010. The Fungicidal Terpenoids and Essntial oil from Litsea cubeba in Tibet. Mol., 15: 7075-7082.

Wei, X.Y., Huang, H.J., Wu, P., Cao, H.L. and Ye, W.H. 2004. Phenolic constituents from Mikania micrantha. Biochemical Systematics and Ecol., 32: 1091-1096.

\section{How to cite this article:}

Pramila Tripathi, Habung Yami and A.K. Shukla. 2016. Evaluation of Antifungal Activity of Artimesia, Litsea and Mikania Essential Oils against Post-harvested Fungal Diseases of Kiwifruits. Int.J.Curr.Microbiol.App.Sci. 5(9): 19-29. doi: http://dx.doi.org/10.20546/ijcmas.2016.509.003 